J. Nonlinear Var. Anal. 4 (2020), No. 1, pp. 1-3

Available online at http://jnva.biemdas.com

https://doi.org/10.23952/jnva.4.2020.1.01

\title{
EDITORIAL
}

\section{A SPECIAL ISSUE DEDICATED TO FRANCO GIANNESSI}

It is our immense pleasure to dedicate this special issue to Professor Franco Giannessi on the occasion of his 85th birthday. Prof. Giannessi's profound and original ideas influenced and shaped various research directions in variational analysis and mathematical optimization over many decades. He pioneered the notion of vector variational inequalities, which is now an important branch of applied mathematics. He independently discovered the powerful mathematical tool called the image space approach, strengthened it, and addressed crucial applications employing it. There are essential results available in optimization, control, equilibrium problems, and variational and quasi-variational inequalities that have been derived by using the mechanism of the image space analysis. He also proposed the notion of the now so-called G-semi-differentiability of a function. This special issue aims to acknowledge and celebrate his beautiful ideas and novel contributions as an innovative researcher of the highest caliber.

This special issue is comprised of ten articles whose contributions are as follows:

G. Mastroeni, M. Pappalardo, and F. Raciti, in the paper "Some topics in vector optimization via image space analysis," provide an excellent introduction to the image space analysis in the context of a vector optimization problem, and its use in proving specific existence results. They also give various separation schemes and discuss their impact on deriving optimality conditions. An exposition to the notion of G-semi-differentiability of a function is also given.

B. Ricceri, in the article "A remark on variational inequalities in small balls," proves new existence results for variational inequalities and the associated Minty formulation given on small closed balls.

The primary focus of the paper, "Nonsmooth dynamics of generalized Nash games" by T. Migot and M. Cojocaru, is to study the generalized Nash equilibrium problem using quasi-variational inequalities in a dynamical system framework. The authors provide a nice introduction to variational inequalities, projected dynamical systems, and equilibrium problems and relate the generalized Nash equilibrium problem to nonsmooth dynamical systems. They provide examples to illustrate the key challenges in handing the underlying variable constraint sets. New existence results are given for the considered dynamical system. The authors propose two classes of algorithms and test them on applied models.

The goal of the work "A projected dynamic system associated with a cybersecurity investment model with budget constraints and fixed demands" by G. Colajanni, P. Daniele, and D. Sciacca is to investigate a network-based cybersecurity investment model with nonlinear budget constraints and fixed demands. The authors use the average value of the security levels for the supply chain network as a weighted 
average by taking into account the flow of commodities, which results in a generalized Nash equilibrium and characterize the equilibrium as a variational inequality. The authors employ the projected dynamic system approach for solving the variational inequalities and provide detailed and convincing numerical results to illustrate the efficacy of the developed framework.

In the paper "Nesterov perturbations and projection methods applied to IMRT," E. Bonacker, A. Gibali, and K-H. Küfer use the simultaneous and cyclic subgradient projection methods with perturbations and apply them to linear and nonlinear optimization problems arising in Intensity-Modulated Radiation Therapy. They provide numerical results to illustrate the mechanism of the perturbations and demonstrate that the approach leads to a significantly faster termination of the algorithm.

The paper "Tensor variational inequalities: theoretical results, numerical methods and applications to an economic equilibrium model" by A. Barbagallo, S. Guarino Lo Bianco, and G. Toraldo, deals with the study of a tensor variational inequality and some projection methods to solve them. In particular, some properties on the solutions to such an inequality are established and a fixed point theorem is proved. Moreover, some numerical methods are introduced and the convergence analysis of them is investigated. A numerical example is also discussed.

N. Hebestreit, in the paper "Algorithms for monotone vector variational inequalities," proposes three algorithms for monotone vector variational inequalities. Depending on the monotonicity assumptions of the underlying map, the algorithms are either based on a projection method or a regularization technique.

The paper "Contingent derivatives of the set-valued solution map of a noncoercive saddle point problem. A Cross-fertilization between variational analysis and inverse problems;" by B. Jadamba, A. A. Khan, M. Sama, and C. Tammer focuses on the inverse problem of parameter identification in a saddle point problem. Under the assumption that the saddle point problem is solvable, the authors study the first-order and second-order contingent derivatives of the set-valued solution map.

The paper "On the existence and approximation of solutions for bi-level vector variational inequalities in Banach spaces," by O. Chadli and R. N. Mohapatra is devoted to the bi-level vector variational inequalities posed in a Banach space setting. The authors provide new existence results by reducing the bi-level vector variational inequalities to a one-level variational problem. Using a new vector auxiliary principle, they propose an iterative algorithm and provide a convergence analysis.

M. A. Mansour, M. A. Bahraoui, and A. El. Bekkali, in their work "A global approximate contraction mapping principle in noncomplete metric spaces" provide an affirmative answer to the question of whether the completeness condition on a metric space can be deleted in deriving an approximate counterpart of the Banach's Contraction mapping principle, which would ensure the existence of $\varepsilon$-fixed points. By relying on the notion of completeness, the authors give new results concerning the existence of an approximate fixed point for contracting set-valued maps in general metric spaces.

In conclusion, we express our most sincere gratitude to all the authors who have contributed to this special issue and to the reviewers who helped us with their thorough reviews.

Rosalind Elster

Halle (Saale), Germany

E-mail address: r.elster@t-online.de 


\title{
Akhtar A. Khan \\ School of Mathematical Sciences \\ Rochester Institute of Technology, Rochester, USA \\ E-mail address: aaksma@rit.edu
}

\author{
Christiane Tammer \\ Institute of Mathematics \\ Martin-Luther-University of Halle-Wittenberg, Halle-Saale, Germany \\ E-mail address: christiane.tammer@mathematik.uni-halle.de
}

